\title{
Preliminary Steps in the Process of Optimization Based on Fingerprint Identification
}

\author{
Maria-Liliana Costin
}

\begin{abstract}
Numerous possibilities of modeling a system of automated fingerprint identification allow us to choose the methods and algorithms that we consider useful in solving a concrete problem at a time. However, the statistical approach and artificial intelligence methods, urges us to use the latest and most useful techniques in the field of fingerprint identification, especially for use in indoor environment. This research focuses on the preliminary steps needed to be done over the fingerprints database, in order to assure an efficient transformation in the steps described below to reach the final scope: an integrated system to be used inside a Prison and/or in conjunction with data from the Police database or for commercial applications.
\end{abstract}

Index Terms-Fingerprints, optimization, statistical methods, artificial intelligence, filters, histogram equalization, global mean, local mean.

\section{INTRODUCTION}

The process of identification based on fingerprints was first done manually and the classification process done by type of details: arches, spirals, circles, divided into groups and subgroups, and a simple search could take weeks or even months. The probability of error was very high and the sampling, classifying and retrieval efforts were tremendous [1]. Automation efforts have resulted in IAFIS Appendix F, since July 1999, but the first use of computers for searching fingerprint files is dating from 1980. The essential condition for the viability of a fingerprint identification system is that the images must contain at least 200 tons of gray and digitized on 8-bit. Although, by date I.A.F.I.S. was an effective system to prevent criminal and terrorist attacks, it will be replaced by NGI Next Generation Identification program, which will make a huge leap in identifying based on fingerprint.

Automatic identification based on fingerprints can be synthesized into five distinct phases, according to Fig. 1: preprocessing (normalization, cutting, alignment, rotation, contrast enhancement, edge detection and transforming into binary image), features extraction (determine the characteristics: arches, whorls, spirals, points of delta), measurement of characteristics (number of details, positioning, angles, distances), classification (according to the details and taking into consideration the variance of the values) and matching (determine the distance between the details compared).

Manuscript received November 21, 2014; revised August 26, 2015.

Maria-Liliana Costin is with the Babes-Bolyai University, Cluj-Napoca, România (e-mail: lilianacostin@yahoo.com).

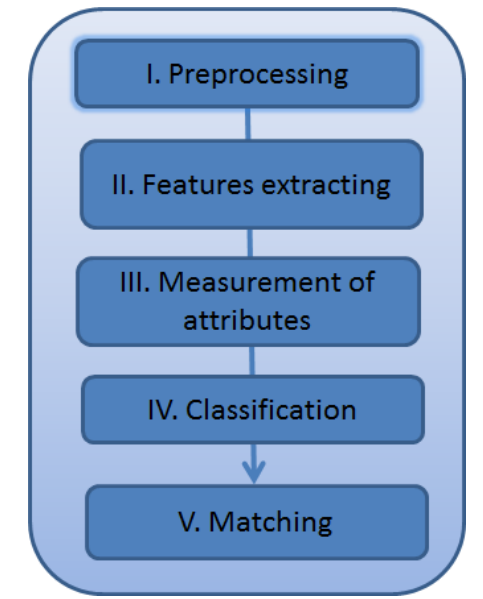

Fig. 1. The 5 stages of the fingerprint identification [2].

\section{STEPS OF FINGERPRINT PROCESSING}

\section{A. Preprocessing Stage}

Preprocessing of fingerprint images seeks to improve image quality by specific algorithms, also known as filters. A brief classification of filters can be as follows [3]-[5]:

- noise reduction / parasites (noise reduction): Median Filter, Filter Olympic, P-Median Filter, Modal Filtering of Classification Results;

- filters optimization quality (Enhancement Filters) [6]: (CS)-Comparison and Selection Filter, WMMR-Med Filter Weighted Majority with Minimum Range -Median, Volter/Unsharp Filter;

- filters texture (Texture): Range Filter, Fourier transform;

- edge detection: smoothing(suppress noise ), enhancement (sharpening), detection (threshold), localization (edge thinning and linking).

\section{B. Extracting Attributes/Details (Feature Extraction)}

Feature extraction includes a wide range of algorithms, uses and [6], [7]:

- algorithms for morphological dilation, erosion, filling, envelope convex skeleton;

- algorithms for image segmentation: detection of discontinuities, connecting segments, determine the contours (Boundaries), global and adaptive filters (threshold) histograms;

- algorithms for representation and description of forms: description contours;

The objectives that need to be reached in this step are:

1) Make "continuity" over the ridge in order to eliminate gaps and to fill spaces due to impurities or imperfections in the scan;

2) Clearer differentiation between ridges and valleys and parallel ridges separating. 


\section{Measuring Attributes}

- $\quad$ statistical methods to minimize loss / risk (conditional equation statistical average risk);

- methods of training, multilayer neural networks, learning algorithms.

\section{Classification}

Pattern classification can be done by:

- statistical discrimination algorithms: Bayes function (Statistical Optimum Bayes Classifier);

- neural networks: training by back propagation;

\section{E. Matching}

The details of each fingerprint are described by multiple attributes: the image location, orientation, type (ridge endings or bifurcation), the image quality in the vicinity of points / details. They "fit" if the distance between them is less than a given tolerance and also less than the tolerance angle.

\section{DifFICULTIES IN THE FINGERPRINT RECOGNITION SYSTEMS}

Automatic recognition of fingerprints is a very difficult problem in terms of the pattern to be found, and this may be due to several factors [4]:

- difference from that same finger can be positioned / scanned in various positions, resulting in a translation thereof;

- rotation of the fingerprint under a certain angle;

- partial overlap in meaning translation and rotation at the same time;

- non-linear deformation, the transition from a three-dimensional image of the finger to two-dimensional, which is due to the malleability of the skin;

- pressure sensor, conditions of the skin, no uniform contact with the sensor finger scanned and other causes such as skin too dry, skin diseases, sweat, moisture, fat. The result is an image with imperfections, which vary from one sampling to the next footprint which makes search more difficult;

- imperfections arising and fingerprinting system, such as excess or lack of ink with which they were obtained;

\section{TYPES OF ERRORS}

Errors in the extraction of details [8] are in turn influenced by the algorithms to extract specific features that are not perfect and produce measurement errors that can arise from several stages of the process:

- separation useful image background image;

- estimate the frequency and orientation of the image;

- detecting the number, types and coordinates of the reference elements;

- detection and post-processing of the details;

- more "aggressive" processing of the algorithms can introduce many differences in location and orientation of the image to grayscale. If poor quality images extracting specific features may introduce false details or to not detect the true details.

\section{COMPARISON OF DIFFERENT PREPROCESSING ALGORITHMS}

\section{A. A Possible Flow-Chart of the First Stage in the Fingerprints Detection Algorithm}

The flow-chart above (Fig. 2) reflects a possible scenario of fingerprint processing in the first stage of the fingerprint identification (Fig. 1), and assumes that the original image needs the global equalization (histogram equalization) and if needed a local equalization (ex. CLAHE-Contrast Limited Adaptive Histogram Equalization-OpenCV ) for contrast enhancement. The Sobel filter (or other like LoG-Laplacian of Gaussian) would be helpful only if the quality of image enhanced before is excellent. Morphological steps are needed after the filtration to determine the affiliation of certain details: if they belong to the pattern or they are simply noise and needs to be dropped. Finally, threshold is used to transform the grey scale image to binary-black and white. In Table I. there are results of algorithms/operations (Fig. 3-Fig. 16) mentioned in the flowchart and obtained with OpenCv routines.

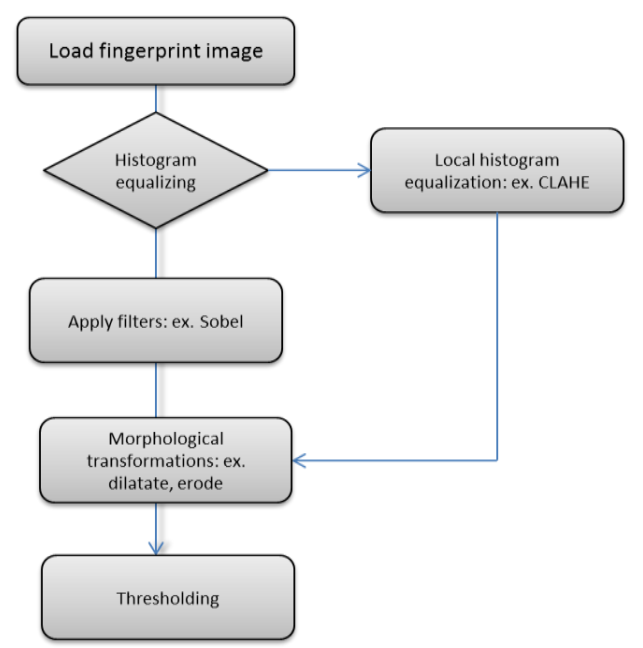

Fig. 2. Flow-chart of the preprocessing stage of the fingerprints.

TABLE I: COMPARISON OF FILTERS APPLYING WITH SEVERAL ALGORITHMS

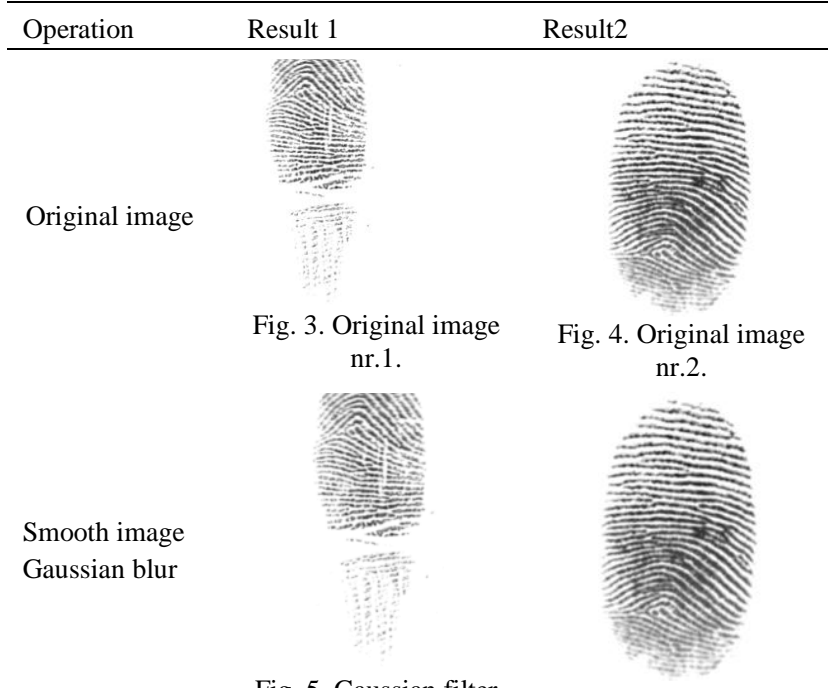

Fig. 5. Gaussian filter applied on image nr.1.
Fig. 6. Gaussian filter applied on image nr.2. 
Edge detection Sobel filter

Morphological Operations

- CLAHE

- thresh holding

- dilate

- erode

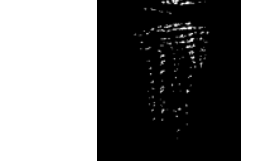

Fig. 15. Erosion applied on image nr.1. (1)

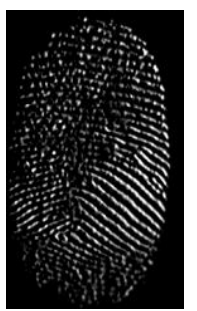

Fig. 8. Sobel filter applied on image nr.2

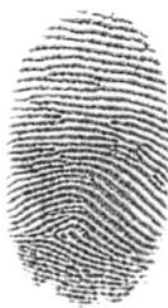

Fig. 9. Morphological transformation applied on image nr.1

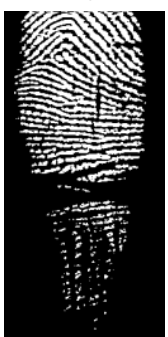

Fig. 11. Thresh holding transformation to binary on image nr.1.

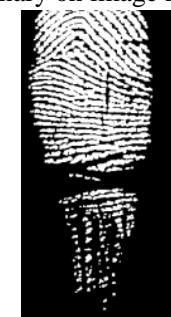

Fig. 13. Dilate transformation on image nr.1.
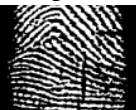
tist

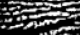

The comparison of processing algorithms reveals the following:

- the histogram equalization is useful but there are better results using locally adaptive histogram equalization like: CLAHE (Fig. 9-Fig. 10) or others not implemented here like L.A.C.E.- Locally Adaptive Contrast Enhancement;

- applying smoothing filters (Fig. 5-Fig. 6) are leading towards reducing the noise and thus to loose unwanted/false details;

- morphological operations (Fig. 13-Fig. 16) are normal steps preliminary to feature extraction and they give reasonably good results;

- thresholds are used for obtaining binary images but also for determining details (Fig. 11 and Fig. 12), and the parameters used in the process are equally important in order to keep essential data and "loose" unwanted/false details;

- Comparison of the results is made by presenting results side-by-side and the use of statistical methods for this task is not very common. In addition we can conclude that is even more difficult to choose a set of parameters that could work perfect on a large set of images.

B. An Heuristic Approach of the First Stage in the Fingerprints Identification Process

The algorithm proposed has two stages:

1) Histogram equalization of images:

Equalizes image histograms for non-cropped images (Fig. 19-Fig. 26) and for cropped images (Fig. 29-Fig. 38) by taking into consideration: global mean $(\mathrm{mg})$ of the entire image, local mean $(\mathrm{ml})$ of each neighborhood of $3 \times 3$ pixels (ml) or even an estimation of an average vicinity like: 2 consecutive details estimated (in pixels) after a calculus applied on randomly chosen columns in the image;

Compares the local mean $(\mathrm{ml})$ of each pixel with the global mean $(\mathrm{mg})$ and if the (local mean<global mean), the pixel is getting a value like: (initial value)*(1-(global mean/ local_mean $)^{*} 1 /$ vicinity) or taking into consideration the number of black/white pixels that compose an standard area (picked by the programmer, for example 2 black details and 1 white or other), in order to make a local equalization of the area;

Tables II and III show a comparison between two approaches, if the algorithm is applied on the original not cropped images and the second on the cropped ones(Fig. 17-Fig. 26 and Fig. 27-Fig. 38); The results show that better resolution and contrast is gained by using the cropped images;

TABLE II: RESULTS OF HEURISTIC ALGORITHM FILTER ON ORIGINAL-NOT CROPPED IMAGES

\begin{tabular}{cccc}
\hline Operation & Result 1 & Result2 \\
Original image &
\end{tabular}

Fig. 17. Original image nr.1.

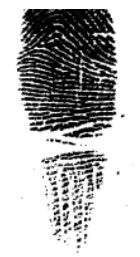

Fig. 19. Initial equalization of histogram of image nr.1.
Fig. 18. Original image nr.2.

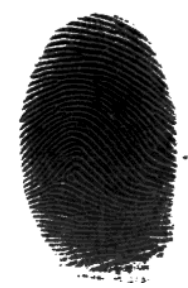

Fig. 20. Initial equalization of histogram of image nr.2. 
Equalize image histogram after heuristic algorithm(step $n+2$ )

Equalize image histogram after heuristic algorithm(step $n+4)$

Equalize image histogram after heuristic algorithm(step $n++)$

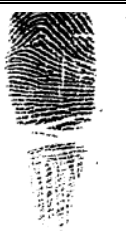

Fig. 21. Equalization of image histogram of image nr. 1 after heuristic algorithm(step $n+2)$.

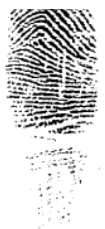

Fig. 23. Equalization of image histogram of image nr.1 after heuristic algorithm(step $n+4)$.

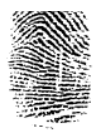

Fig. 25. Equalization of image histogram of image nr. 1 after heuristic

algorithm(step $n++)$.

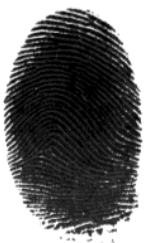

Fig. 22. Equalization of image histogram of image nr.2 after heuristic algorithm(step $n+2$ ).

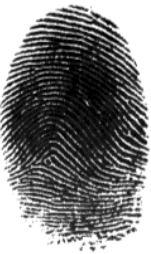

Fig. 24. Equalization of image histogram of image nr.2 after heuristic

algorithm(step $n+4)$.

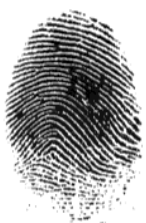

Fig. 26. Equalization of image histogram of image nr.2 after heuristic algorithm(step $n++$ ).

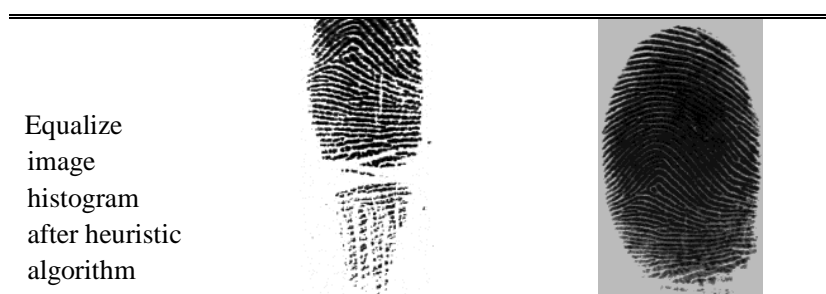

(step $n+2$ )

Fig. 33. Equalization of image nr.1 histogram after applying step $n+2$ of heuristic algorithm.

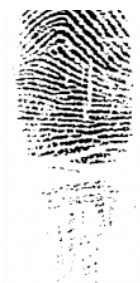

Fig. 35. Equalization of image nr.1 histogram after applying step $n+4$ of heuristic algorithm.

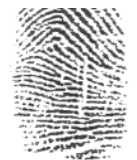

image

histogram after heuristic algorithm

(step $n++)$

\section{Fig. 37. Equalization of image nr.1 histogram after applying step $n++$ of heuristic algorithm.}

Fig. 34. Equalization of image nr.2 histogram after applying step $n+2$ of heuristic algorithm.

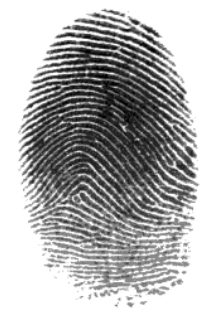

Fig. 36. Equalization of image nr.2 histogram after applying step $n+4$ of heuristic algorithm.

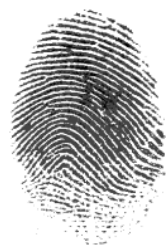

Fig. 38. Equalization of image nr.2 histogram after applying step $n++$ of heuristic algorithm.

TABLE III: Results OF HeURistic Algorithm FILTER CROPPED IMAGeS

\begin{tabular}{lll}
\hline \hline Operation & Result 1 & Result2 \\
\hline & &
\end{tabular}

Fig. 27. Original image nr.1.

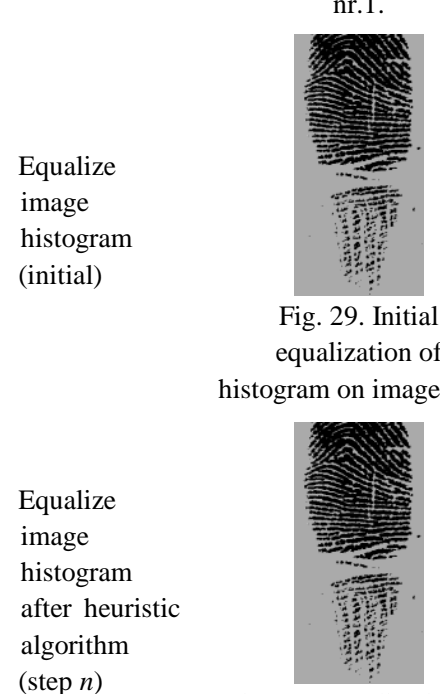

Fig. 31. Equalization of histogram on image nr.1 after applying step $n$ of heuristic algorithm.
Fig. 28. Original image nr.2.

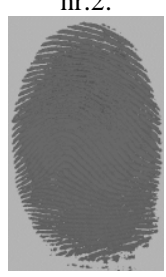

Fig. 30. Initial equalization of histogram on image nr.2.

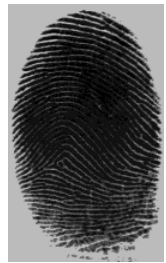

Fig. 32. Equalization of histogram on image nr.2 after applying step $n$ of heuristic algorithm.
A closer look at the two images after Sobel operator convolution (Table IV, Fig. 39-Fig. 40) shows that the details can be emphasized more as long with suppressing the "noise" (false details).

TABLE IV: RESULTS OF THE SOBEL OPERATOR ON THE CROPPED IMAGES

\section{Result of Sobel operator on}

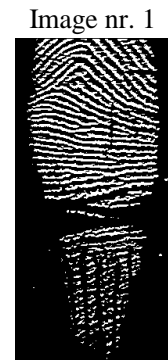

Fig. 39. Image nr.1 after Sobel operator
Result of Sobel operator on

Image nr. 2

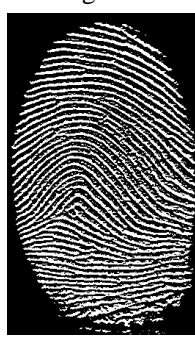

Fig. 40. Image nr.2 after Sobel operator
The resulting image in "binary" black and white, it corresponds to the scope, but the images are noisy, they have a lot of false details, it needs to be preprocessed further.

2) The second step processes the fingerprint images as following:

a) Determines the matrix corresponding to the grey scale pixels;

b) Estimates the value of details: how many pixels correspond to details and how many to the white spaces 
between them:

- choosing the sample of rows from the initial matrix, $40 \%$ of the columns randomly chosen;

- $\quad$ using the gradient method on each column and compute the total pixels, the white and the black;

- estimate how many details ("white space" and "black space") there are in the column and on the sample;

c) Those details will be used to divide the image, in local areas according to the parameters above, for instance: each area or vicinity is a square with the side equal to 1 "black space"+1 "white space";

d) Computes the local mean of pixels for each area/vicinity and stores it in an initial matrix;

e) Computes in each vicinity the maxima and minima, excluding the values 0 and 255 , so it results a gradual process of "moving" the pixels closer to the lower and higher threshold to the corresponding extremes, they become 0 or 255 ;

f) The local mean matrix is recalculated and the process is reiterated until the local vicinity extrapolates sufficient to result after convolution a black and white image with less noise.

TABLE V: RESUlTS OF HEURISTIC ALGORITHM PROCESSING APPLIED ON CROPPED IMAGES AND THE TRANSFORM TO "BINARY"

Original image $\quad$ Processed image $\quad$ Sobel convolution result

Fig. 41. Original image $\mathrm{nr} .2$.

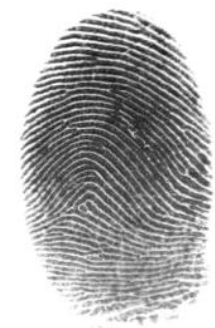

Fig. 44. Original image nr. 1 .
Fig. 42. Processed image nr.2.

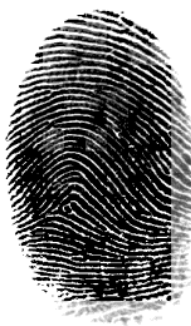

Fig. 45. Processed image nr.1.
Fig. 43. Sobel convolution result on image nr. 2 .

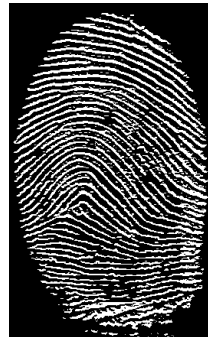

Fig. 46. Sobel convolution result on image nr. 1.
The process of filtering the digital images is an important stage in the identification algorithm because the clarity of the resulting image determines the success of the later steps.

In the Table $\mathrm{V}$, we have a quick overview about the important steps of the initial images (Fig. 41, Fig. 44) to the processed ones (Fig. 42, Fig. 45) with the heuristics algorithm and finally to binary image (Fig. 43, Fig. 46).

In Table VI are illustrated the histograms corresponding to the same image in different stages of processing: the original image (Fig. 47), the equalized image histogram in stage 1 (Fig. 48) and the histogram of the resulting image after stage 2 (Fig. 49). The "migration "of the grey tones to the 0 and 255 is obvious and responds to the scope of this phase of the fingerprint identification system.

TABLE VI: DYNAMICS OF THE GREY TONES VALUES DURING THE LOCAL EQUALIZATION ALGORITHM FOR THE CROPPED IMAGES

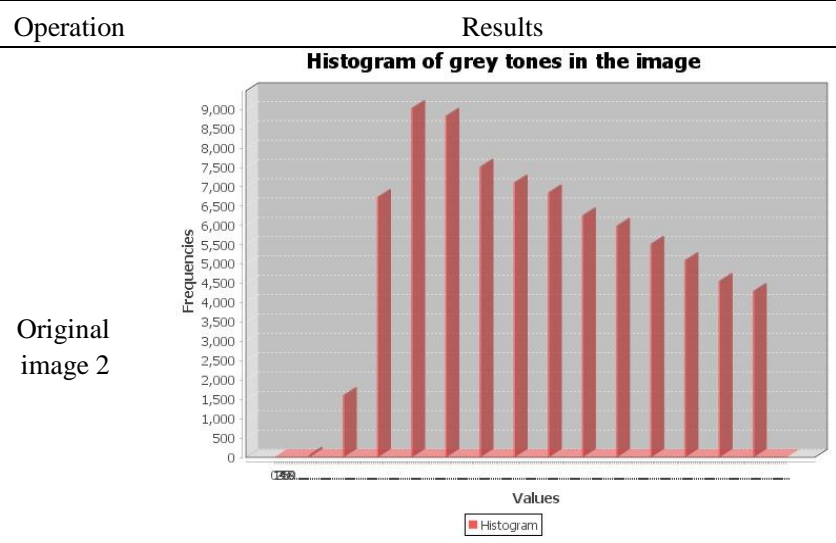

Fig. 47. Histogram of grey tones in the original image nr.2 (corresponding to Fig. 28)

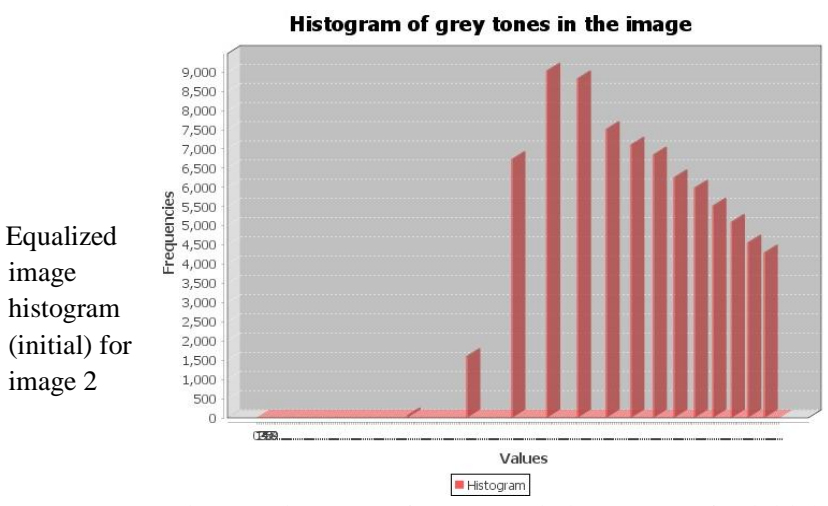

Fig. 48. Histogram of grey tones in image nr.2 after initial equalization.

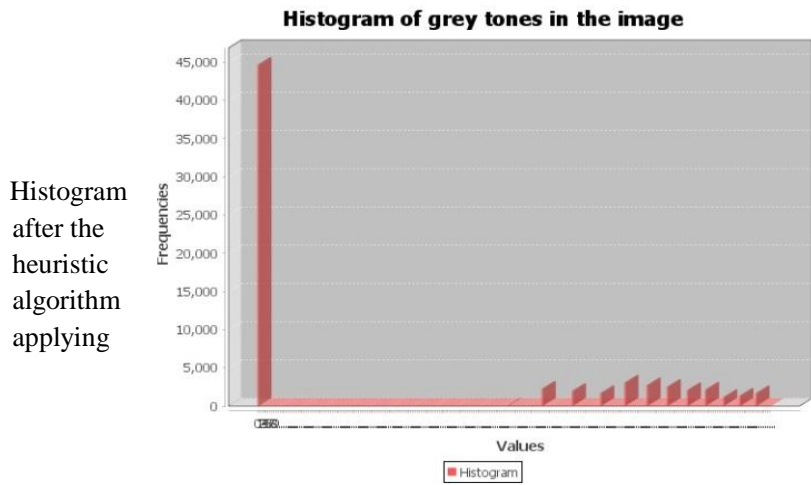

Fig. 49. Histogram of grey tones in image nr.2 after applying the heuristic algorithm.

Future directions about the current algorithm are:

- determining the optimum number of steps in the process taking into consideration another type of means, besides the global mean and local mean;

- in order to determine the variations of the pixel values in a larger area, to determine a customized local vicinity (other than $3 \times 3$ used in this case), or the estimated from stage 2, step b, taking into consideration the thickness of the details and the correlation between them;

- applying other filters for obtaining more accurate images;

- determine an automated way of choosing the best filter for each image; 
- obtaining the "skeleton" of the image and determine the features for comparison.

\section{ACKNOWLEDGMENT}

This work was possible with the financial support of the sectorial operational program for human resources development 2007-2013, co-financed by the European social fund, under the project number POSDRU/ 159/1.5/S/132400 with the title "Young researchers of success - professional development in the interdisciplinary and international context”.

\section{REFERENCES}

[1] M. L. Costin, Biometric Identification Technology, Tehnologia Identificării Biometrice, RO: Lumen, 2013, pp. 21-28.

[2] I. Ispas, "The image recognition and classification, a four-step modeling", in Proc. The 2nd International Conference on European Integration -Between Tradition and Modernity, Tirgu Mures, 2007, pp. 124-132.

[3] J. Wayman, A. Jain, D. Maltoni, and D. Maio, Biometric Systems-Technology, Design and Performance Evaluation, USA: Springer, 2005, pp.24-59.

[4] L. Hong, Y. Wan, and A. K. Jain, "Fingerprint image enhancement: algorithms and performance evaluation," IEEE Trans. Pattern
Analysis and Machine Inteligence, vol. 20, no. 8, pp. 777-789, August, 1998.

[5] D. Maltoni, D. Maio, A. K. Jain, and S. Prabhabar, Handbook of Fingerprint Recognition, 2nd ed. London, U.K.: Springer, 2009, pp. 249-315.

[6] T. Khana, M. A. U. Khanb, and Y. Konga, "Fingerprint image enhancement using multi-scale DDFB based diffusion filters and modified Hong filters," International Journal for Light and Electron Optics, vol. 125, no. 16, pp. 4206-4214, August 2014.

[7] J. M. Guoa, Y. F. Liua, J. Y. Changa, and J. D. Lee, "Fingerprint classification based on decision tree from singular points and orientation field," Expert Systems with Applications, vol. 41, no. 2, pp. 752-764, February 2014.

[8] S. Bleay, D. Charlton, Fingerprint Identification, Encyclopedia of Criminology and Criminal Justice, New York, U.S.A.: Springer, 2014 pp. 1648-1664.

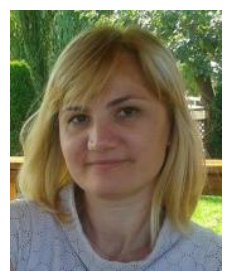

research projects.
Maria-Liliana Costin was born in Romania. She got a $\mathrm{PhD}$ degree in cybernetics and economic statistics in 2009. Currently she is a postdoctoral researcher at University Babes-Bolyai in Cluj-Napoca, in the field of Informatics.

She is the author of papers published in international journals. She is also a reviewer at several international publications and evaluator in the field of 